\title{
THE PROCESSING OF IMAGE DATA COLLECTED BY LIGHT UAV SYSTEMS FOR GIS DATA CAPTURE AND UPDATING
}

\author{
N. Yastıkl1, I. Bagci and C. Beser
}

\begin{abstract}
YTU, Civil Engineering Faculty, Department of Geomatics Engineering, 34210 Davutpasa, Istanbul, Turkey ynaci@yildiz.edu.tr, irfanbagcioglu@gmail.com, cihanbeser@gmail.com
\end{abstract}

Commission II, III, IV \& VII Joint Meeting

KEY WORDS: UAVs, Photogrammetry, Sensor, Orientation, DEM, orthoimage, GIS

\begin{abstract}
:
The collection and updating of 3D data is the one of the important steps for GIS applications which require fast and efficient data collection methods. The photogrammetry has been used for many years as a data collection method for GIS application in larger areas. The Unmanned Aerial Vehicles (UAV) Systems gained increasing attraction in geosciences for cost effective data capture and updating at high spatial and temporal resolution during the last years. These autonomously flying UAV systems are usually equipped with different sensors such as GPS receiver, microcomputers, gyroscopes and miniaturized sensor systems for navigation, positioning, and mapping purposes. The UAV systems can be used for data collection for digital elevation model DEM and orthoimages generation in GIS application at small areas. In this study, data collection and processing by light UAV system will be evaluated for GIS data capture and updating for small areas where not feasible for traditional photogrammetry. The main aim of this study is to design the low cost light UAV system for GIS data capture and update. The investigation was based on the aerial images which recorded during the flights performed with UAV system over the test site in Davutpasa Campus of Yildiz Technical University, Istanbul. The quality of generated DEM and ortho-images from UAV flights was discussed for GIS data capture and updating for small areas.
\end{abstract}

\section{INTRODUCTION}

Unmanned Aerial Vehicles (UAVs) supplies new opportunities in remote sensing and photogrammetry applications as well as applications in robotics and computer vision (Eisenbeiss 2009; Evaraerts 2009; Niethammer et al., 2009; Irschara et al., 2010; Sauerbier et al., 2010). In recent years, UAV-Systems gained attraction in geosciences because of the possibility of capturing cost effective data at high spatial and temporal resolution. The light UAV platform with a control system, which is combined with amateur or SRL digital cameras and GNSS/INS systems, can be used for photogrammetric data capture. The limiting factors of the UAVs are their small size and reduced pay-load which is limiting the transportation of the high quality IMU devices coupled with the airborne cameras or LiDAR sensors used for mapping. The low cost UAV system can be build up at a very reasonable price in comparison to the digital photogrammetric systems. Since the GIS applications require updating 3D geodata with fast and efficient surveying method, the UAV systems can be used for data capture and updating. The DEM and ortho-images are the indispensable geodata data of the GIS application which can be produced with UAVs in very reasonable price especially at areas of limited extent.

The main aim of this study is to design the low cost light UAV system for GIS data capture and update. The performance evaluation of designed low cost light UAV system is also intended for DEM and ortho-images generation over the test site in Davutpasa Campus of Yildiz Technical University, Istanbul.

\section{METHODOLOGY}

\subsection{UAV description}

As mentioned earlier, we designed the octocopter with eight propellers as a low cost light UAV system for GIS data capture and update. The designed UAV system is consist of the airframe, motors, actuators and power supply. The used frame contains the aluminum square tubes and carbon fiber base plates. The landing gear was made of carbon fiber tube. The system was powered by brushless motors and lithium polymer battery (Figure 1).

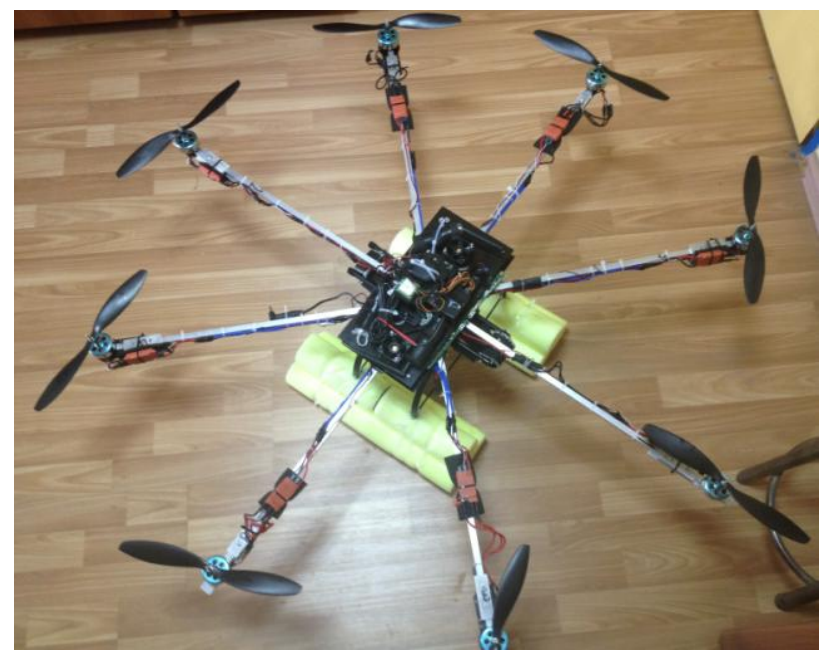

Figure 1. The designed low cost light UAV systems 
The ArduFlyer V2.5 flight control board controls sensor fusion and current flight information by applying three axis gyroscopes, six DOF accelerometers, digital compass sensor and a barometer. The Ublox Neo 6P (PPP) GPS was integrated on designed UAV system. The UAV systems has auto take off, land and autonomous flight properties and capable of performing programmed GPS missions with waypoints. A Turnigy 9x remote control sends commands within the $2.4 \mathrm{GHz}$ frequency band that subsequently is processed by the Flight Control board. A bidirectional solution works within $433 \mathrm{MHz}$ provided by radio links. This radio links send flights data to ground control station. The camera mount which was constructed from fiberglass, aluminum alloy and high speed servo motor was used to provide pan, tilt and roll control of camera during the flight. The Canon SX 150 compact camera has been chosen because of the lightweight and manual functions. The take of weight of the designed UAV was $5 \mathrm{~kg}$ (with camera) and flight time was approximately 15 minutes. The open source Mission Planner software (DIYDrones, 2013) was used for the flight planning. The overall cost for the designed UAV system was about 1000 Euro.

\subsection{Description of the test area and data acquisition}

The small test side in Davutpasa Campus of Yildiz Technical University, Istanbul was used for performance evaluation of the designed UAV system (Figure 2). The main motivation was the test the low cost UAV system performance for GIS data capture and updating for small areas.

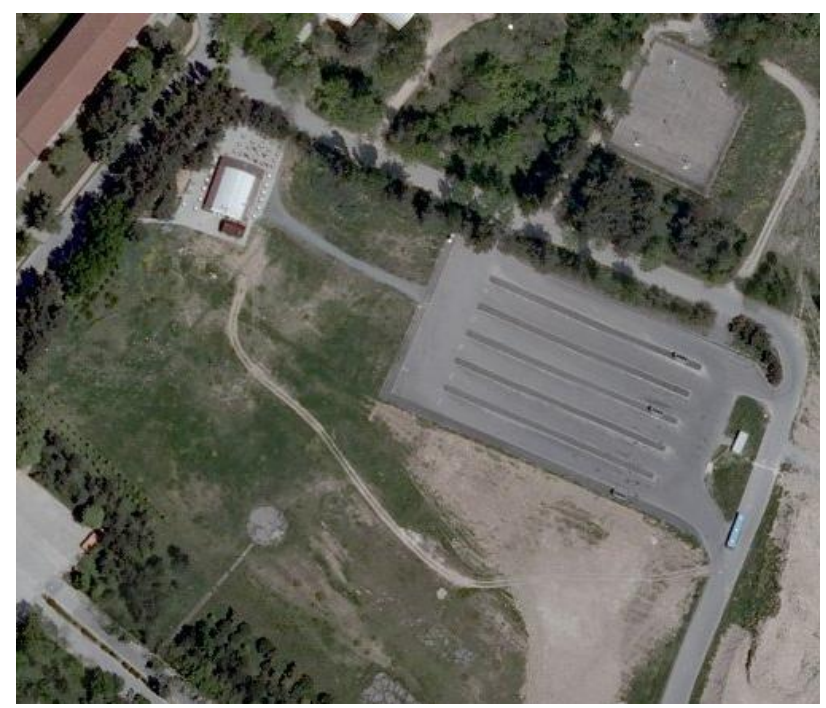

Figure 2. The overview of the test side in Davutpasa Campus of Yildiz Technical University, Istanbul

The data acquisition has been performed on 26 August 2013 with designed UAV systems. The small block was planned which consist of 20 images including two flight strips with $60 \%$ endlap in flight direction and 60\% sidelap (Figure 3). The 45 ground control points (GCP) were determined in reference coordinate system using differential GNSS measurements. The images were recorded over the test area with the Canon SX 150 compact camera (4.89 mm lens, $15 \mathrm{Mpx}$ and pixel size 1.42 $\mu \mathrm{m})$ at the ground sample distance (GSD) of $1.4 \mathrm{~cm}$ (Figure 4).

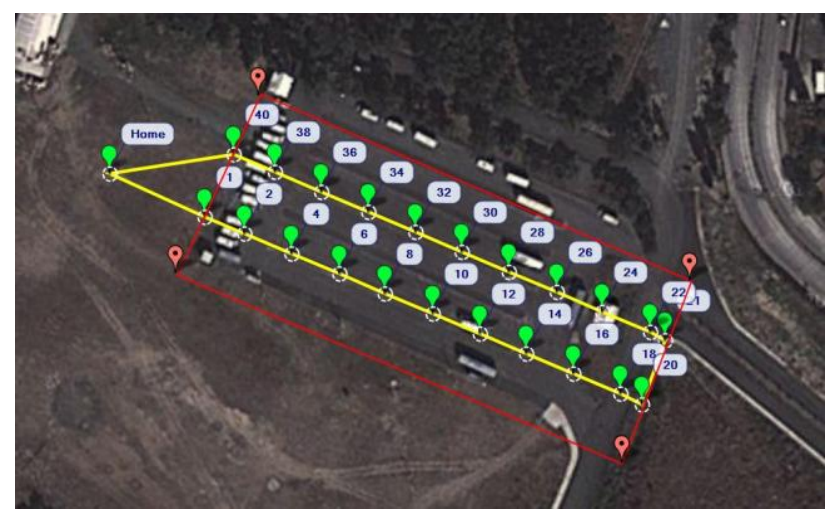

Figure 3. The planned flight lines and camera stations

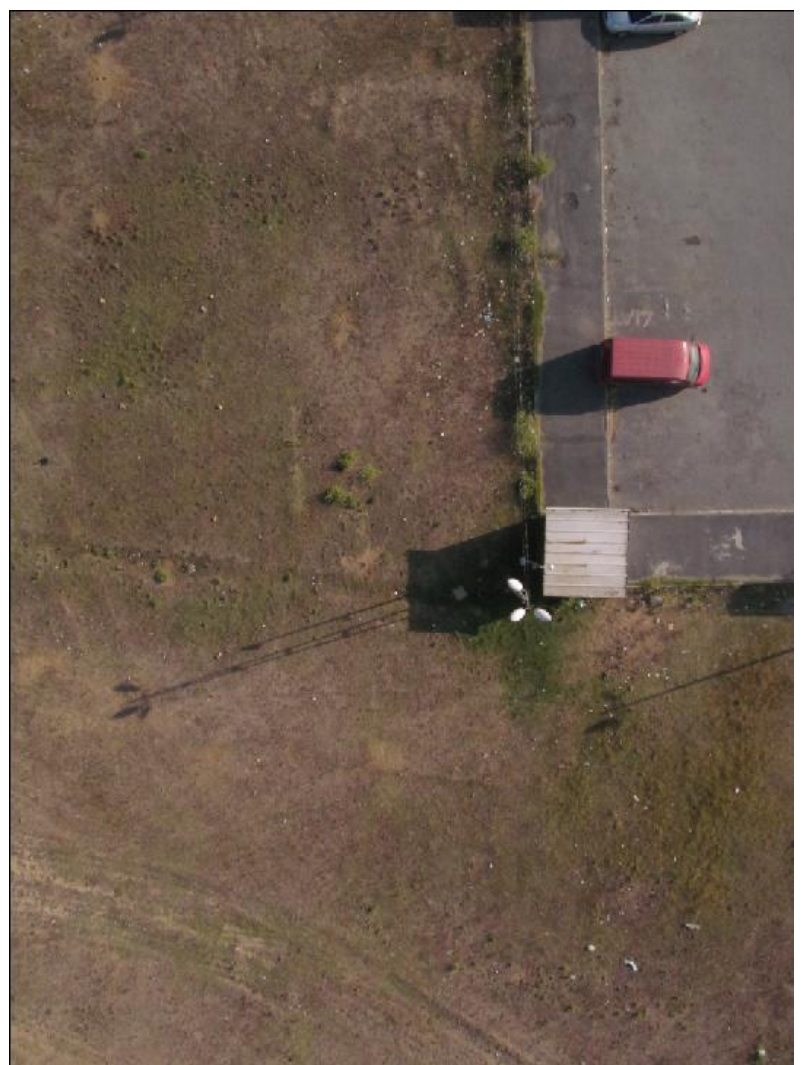

Figure 4. The recorded image of test area acquired with Canon SX 150 compact camera mounted on designed UAV systems

\section{PHOTOGRAMMETRIC PROCESSING}

\subsection{Camera calibration}

The geometric calibration of Canon SX 150 compact camera was accomplished with a 3D test object with 77 control points distributed at different depths (Figure 5) using PHIDIAS software which was developed by PHOCAD. The geometric calibration parameters including the position of principal point and focal length (x0, y0, c), radial distortion $(\mathrm{K} 1, \mathrm{~K} 2, \mathrm{~K} 3)$ and tangential distortion (P1, P2) parameters were determined with the bundle block adjustment. The statistical significance of introduced geometric calibration parameters was checked. 


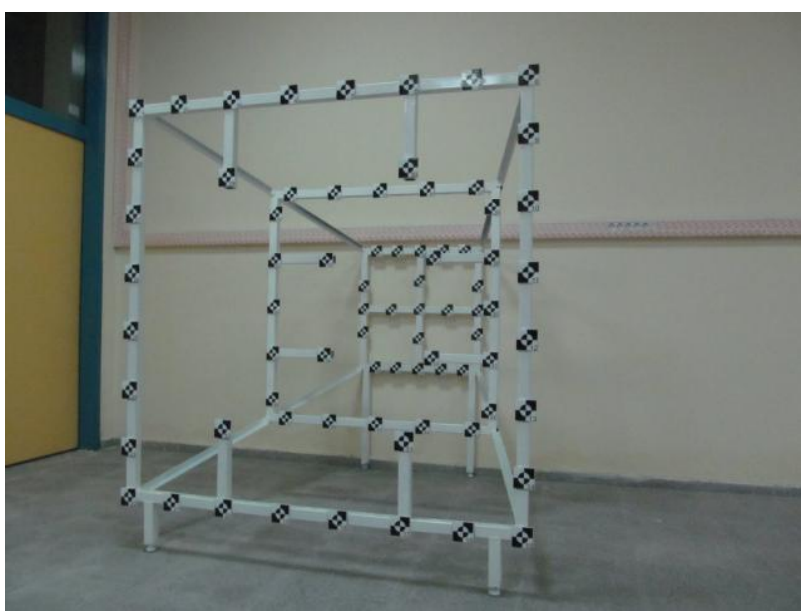

Figure 5. 3D test object for geometric camera calibration

\subsection{Aerial triangulation}

The ZI ImageStation Automaic Triangulation software was used for aerial triangulation. The image measurements were performed manually for tie and pass points' measurement. The image coordinate of the control points were measured on the images. The Figure 6 shows the measured tie points, pass point and control points on the images. The obtained sigma-naught of was $4.815 \mu \mathrm{m}$ as a results of the bundle block adjustment performed with The ZI ImageStation Automaic Triangulation software. The computed RMSE on 45 ground control points were $1.4 \mathrm{~cm}, 1.7 \mathrm{~cm}$ and $2.3 \mathrm{~cm}$ in the $\mathrm{X}, \mathrm{Y}$, and $\mathrm{Z}$ directions, respectively. The obtained result verified that high accuracy can be achieved for image orientation with small format low cost camera after proper geometric calibration. The stereo model generation has been performed without any problem after aerial triangulation.

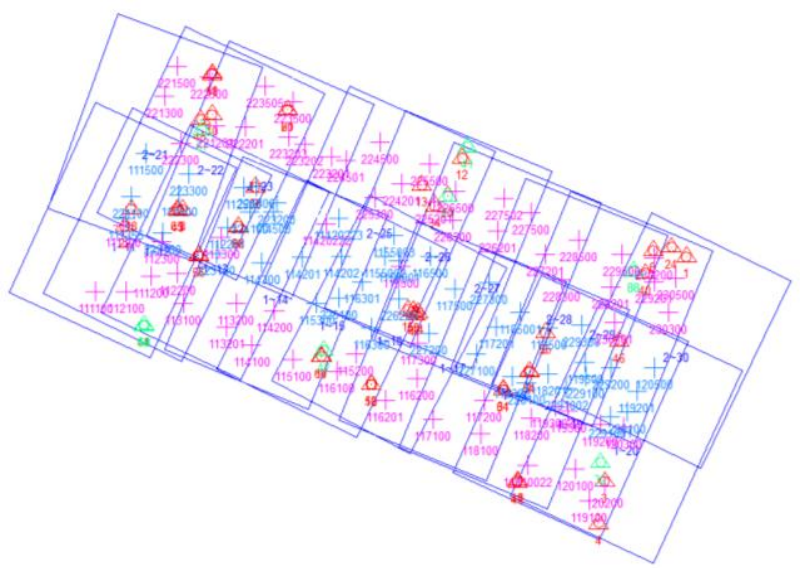

Figure 6. The measured tie points, pass point and control points on the image block

\subsection{DEM and Orthoimage generation}

The ZI ImageStation Automatic Elevations software has been used for the high resolution DEM generation. The DEM generations for each stereo model have been performed separately. The image matching for automatic DEM generation has been performed on green channel. The Figure 7 shoes the generated DEM of the test side.

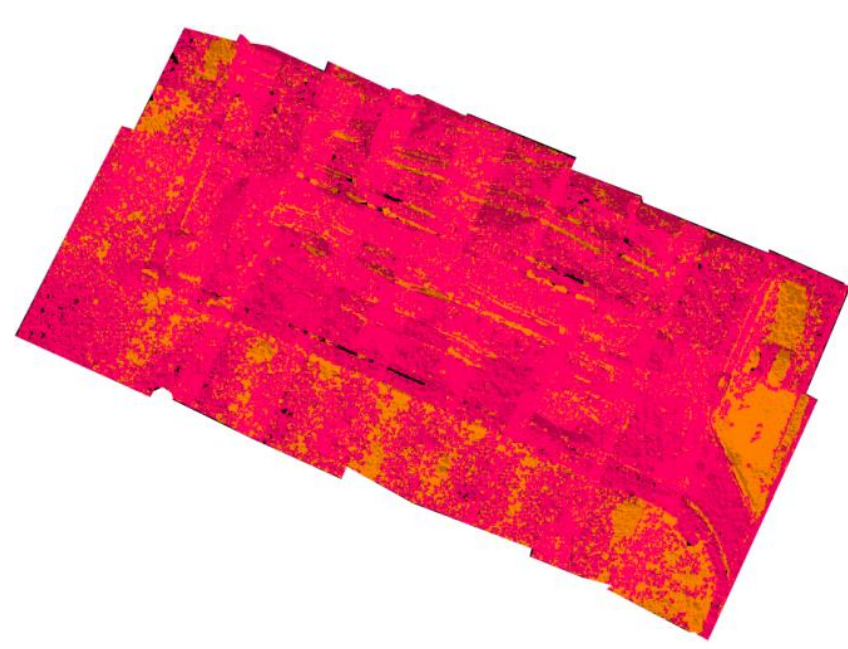

Figure 7. The generated DEM of the test side

The ortho images were generated with Erdas LPS module with the ground sample distance of $2 \mathrm{~cm}$ using the all recorded image with UAV system. The mosaic image which covers all test side with the GSD of $2 \mathrm{~cm}$ was generated (Figure 8).

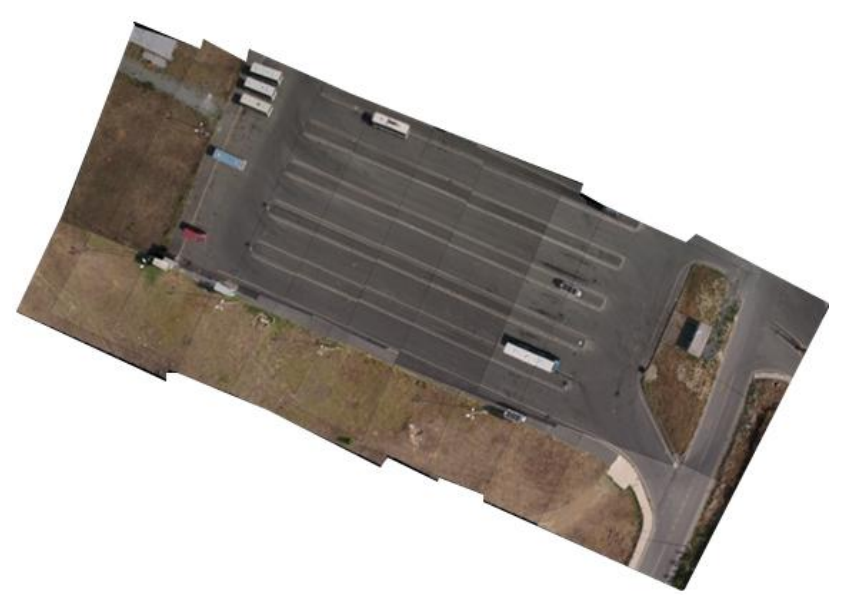

Figure 8 . The mosaic image of the test side

\section{CONCLUSION}

In this paper, the performance of the designed low cost light UAV system was tested for GIS data capture and updating. The overall cost of the designed UAV system was about 1000 Euro. The open source software was used for the flight planning. The automatic take off, land and autonomous flight were performed by programmed GPS missions with waypoints.

The geometric camera calibration was performed successfully which was the key element for accurate aerial triangulation. The achieved high accuracy for the aerial triangulation confirmed that the orientation of the images can be performed successfully which recorded by the low cost camera mounted on UAV system. The high numbers of GCPs were used since the precise camera projection center coordinate were not obtained properly during the flight mission. The dense image matching failed in some areas of our test side during the automatic DEM generation but orthoimage generation was performed without any problem. Since the high quality reference data sets such as LiDAR data was not available on test side, the detailed performance evaluation of generated DEM was not performed. 
The obtained results from our test side verified that the low cost light UAV system can be used for the DEM and ortho-images generation in GIS application for small areas where not feasible for traditional photogrammetry.

\section{REFERANCES}

DIYDrones: www.diydrones.com (accessed 30 September, 2013)

Eisenbeiss, H., 2009. UAV Photogrammetry. DISS. ETH NO. 18515.

Everaerts, J., 2009. NEWPLATFORMS - Unconventional Platforms (Unmanned Aircraft Systems) for Remote Sensing 102 pages. Frankfurt a.M. EuroSDR official publication 56.

Irschara, A., Kaufmann, V., Klopschitz, M., Bischof, H., Leberl, F., 2010. Towards fully automatic photogrammetric reconstruction using digital images taken from UAVs. Proc. ISPRS Symposium, 100 Years ISPRS - Advancing Remote Sensing Science.

Niethammer, U., Rothmund, S. and Joswig, M., 2009. UAV-based remote sensing of the slow moving landslide Super-Sauze, In: Landslide processes, CERG Editions, Strasbourg, pp. 69-74.

Sauerbier, M., Eisenbeiss, H., 2010: UAVs for the documentation of archaeological excavations. IAPRS\&SIS, Vol. 38(5), Newcastle upon Tyne, UK. 